\title{
DISEÑO Y VALIDACIÓN DE UNA ESCALA DE AUTOEVALUACIÓN DE COMPETENCIAS DIGITALES PARA ESTUDIANTES DE PEDAGOGÍA
}

\section{DESIGN AND VALIDATION OF A SCALE OF SELF-ASSESSMENT DIGITAL SKILLS FOR STUDENTS OF EDUCATION}

\author{
Dra. Carolina Flores-Lueg ${ }^{1}$ \\ cflores@ubiobio.cl \\ Dra. Rosabel Roig Vila ${ }^{2}$ \\ Rosabel.roig@ua.es \\ ${ }^{1}$ Universidad del Bío-Bío. Facultad de Educación y Humanidades. Dpto. de Ciencias de la \\ Educación. Avda. La Castilla s/n, Chillán (Chile). \\ ${ }^{2}$ Universidad de Alicante. Facultad de Educación. Dpto. de Didáctica General y \\ Didácticas Específicas. Ap. Correos 99, 03080, Alicante (España).
}

Este trabajo presenta el proceso de validación de una Escala de Autoevaluación de competencias digitales para estudiantes de Pedagogía. La estructura del instrumento contempla cinco dimensiones: Pedagógica - Social, Ética y Legal - Técnica - Gestión Escolar y Desarrollo Profesional. El procedimiento consideró la validación de contenido, validación de constructo a través del Análisis Factorial y la determinación de fiabilidad a través del alfa de Cronbach. La escala se aplicó a una muestra de 161 estudiantes de Pedagogía de la Universidad del Bío-Bío (Chile) que habían comenzado la actividad de práctica profesional. El proceso desarrollado permitió dar validez científica al instrumento diseñado.

Palabras claves: Educación Superior; Formación preparatoria de docentes; competencia digital; autoevaluación.

This paper presents the process of validation of a self-rating scale digital skills for students of Pedagogy. The structure of the instrument includes five dimensions: Pedagogical - Social, Ethical and Legal - Technical - School Management and Professional Development. The validation procedure considered the content, construct validation through factorial analysis and determination of reliability through Cronbach's alpha. The scale was applied to a sample of 161 students of Pedagogy of the University of the Bio Bio (Chile) who had started the activity of professional practice. The process developed allowed to give scientific validity to the instrument designed.

Key words: Higher Education; Preparatory training of teachers; digital competence; selfevaluation.

Píxel-Bit. Revista de Medios y Educación. No 48 Enero 2016. ISSN: 1133-8482. e-ISSN: 2171-7966. doi: http://dx.doi.org/10.12795/pixelbit.2016.i48.14 


\section{Introducción.}

Uno de los grandes retos que está enfrentando la educación superior corresponde a la formación de profesionales capaces de participar activa y responsablemente en todos los ámbitos de la sociedad, siendo eficientes y creativos en el desempeño de sus funciones, junto con dar respuesta a las necesidades productivas, a la innovación tecnológica y participar activamente en escenarios cada vez más globalizados. En este contexto, se busca que las y los egresados sean «competentes en el dominio de ciertos códigos y en los nuevos sistemas simbólicos, además, capaces de interactuar con la información disponible en formato digital y a través de las redes de comunicación» (Arras, Torres \& García, 2011, p.4). Por tal razón, la competencia digital está adoptando cada vez mayor relevancia.

Al analizar algunas de las definiciones disponibles en la literatura sobre competencia digital se observa que en todas ellas se combinan principalmente elementos vinculados a la alfabetización informacional, al dominio técnico de herramientas tecnológicas y digitales; algunas también incorporan aspectos cognitivos y comunicativos, mientras que otras consideran los aspectos éticos y legales (Comisión Europea, 2006; Gisbert, Espuny \& González, 2011; Gutiérrez, 2014). Por consiguiente, se puede desprender que la competencia digital engloba un conjunto de otras competencias o subcompetencias (Adell, 2010; Cabero \& Marín, 2014).

En base a lo anterior, entendemos que la competencia digital está referida a la movilización de aquellas habilidades y destrezas que permiten buscar, seleccionar críticamente, obtener y procesar información relevante haciendo uso de las TIC para transformarla en conocimiento, al mismo tiempo, que se es capaz de comunicar dicha información a través de la utilización de diferentes soportes tecnológicos y digitales, actuando con responsabilidad, respetando las normas socialmente establecidas y aprovechando estas herramientas para informarse, aprender, resolver problemas y comunicarse en distintos escenarios de interacción (Flores, 2014).

Al transferir el concepto de competencia digital anterior a la labor del profesorado, nos encontramos con una cuestión que no es fácil de afrontar, porque este actor debe ser capaz no solo de desarrollar la competencia digital en cada una de sus dimensiones, sino que al mismo tiempo se espera que articule dichos conocimientos con sus saberes pedagógicos y disciplinarios, de tal manera que logre ejercer una acción didáctica con ellas, que comprenda que las TIC están a su servicio y que pueden contribuir a renovar las metodologías de enseñanza.

En lo que respecta a la definición de aquellos saberes que conforman la competencia digital docente, en la literatura se pueden encontrar algunas propuestas que proporcionan ciertas orientaciones sobre los conocimientos, habilidades, destrezas y actitudes que debe lograr desarrollar un docente para hacer frente de manera óptima a la incorporación de las TIC dentro del proceso de enseñanza-aprendizaje (Cabero \& Marín, 2014; Gutiérrez, 2014; Prendes, 2010). Asimismo, hoy día encontramos diversas alternativas de Estándares en TIC para el profesorado, las que definen competencias, indicadores y niveles de logro. En este trabajo, entendemos que los estándares pueden ser utilizados como «instrumentos de evaluación del desempeño individual en tareas o 
funciones de especial interés» (Climènt, 2010, p.93), y en este sentido los estándares en TIC corresponderían a un patrón o un modelo de referencia que permite valorar los niveles de desempeño que poseen los docentes o futuros docentes respecto a las distintas dimensiones de la competencia digital. Asimismo, sirven de guía para las universidades en sus programas de formación inicial de maestros (MINEDUC, 2006, 2008; Prendes \& Gutiérrez, 2013; UNESCO, 2008). Sumado a ello, para Mengual y Roig (2012), estas propuestas simbolizan «la voluntad de creación de una taxonomía que permita comprender la forma en cómo las TIC han penetrado en la sociedad y qué elementos son susceptibles de estudio y aprendizaje» (p.27).

Una de las propuestas de estándares específica para la etapa de formación inicial del profesorado, corresponde a los Estándares TIC para Formación Inicial Docente (FID) (Ministerio de Educación de Chile [MINEDUC], 2006,2008). Esta alternativa contiene 16 competencias agrupadas en cada una de las siguientes dimensiones: Pedagógica, Técnica, Social, Ética y Legal, Gestión y Desarrollo Profesional. Al mismo tiempo, presenta un total de 76 indicadores, donde claramente el aspecto Pedagógico es el que presenta un mayor peso, pues integra siete competencias, representando aproximadamente a un $44 \%$ del total de competencias definidas.

Los Estándares TIC para la FID chilenos los hemos seleccionado intencionalmente, no sólo porque son plenamente pertinentes al contexto de estudio considerado, sino porque tenemos la firme convicción de que el desarrollo de la competencia digital docente es un desafío que debe ser abordado desde el nivel de formación inicial, pues como señala
De Saint (2008), la formación inicial docente debe hacerse cargo de las nuevas formas de aprender y de enseñar del Siglo XXI. En esta misma línea diversos autores (Brun, 2010; Flores, 2014; Vaillant, 2013) sostienen que los planes de formación de los futuros docentes deben considerar la integración e implementación efectiva de las TIC en los procesos formativos, ya que ésta sería la instancia propicia para que los futuros docentes egresen siendo competentes tanto en conocimientos pedagógicos y disciplinares, como en conocimientos sobre las TIC aplicadas a la educación con fines didácticos.

A partir de todas estas consideraciones, nos propusimos como objetivos de investigación:

- Diseñar un instrumento de autoevaluación sobre competencia digital específico para estudiantes de Pedagogía.

Determinar la validez de contenido de la escala de autoevaluación de competencia digital para estudiantes de Pedagogía elaborada.

Establecer la validez de constructo y fiabilidad de la escala de autoevaluación de competencia digital para estudiantes de Pedagogía.

\section{Metodología.}

\subsection{Procedimiento.}

Se diseñó un instrumento ad hoc para el estudio, denominado Escala de Autoevaluación de Competencia digital para estudiantes de Pedagogía, para ello se consideraron las siguientes fases:

- Revisión de algunas escalas sobre competencia digital del profesorado disponibles en la literatura (Area, 2010; 
Cabero, Llorente \& Marín, 2010; García, 2011; Prendes \& Castañeda, 2010). Además, se analizaron y contrastaron las siguientes propuestas de estándares TIC para docentes: NETS-T (ISTE, 2008), Normas de Estándares TIC (UNESCO, 2008) y Estándares TIC para la Formación Inicial Docente (FID) (MINEDUC, 2006, 2008).

A partir de los análisis anteriores se formularon 106 ítems iniciales de la escala, agrupados en las siguientes dimensiones: Aspectos Pedagógicos; Aspectos Sociales, Éticos y Legales; Aspectos Técnicos; Gestión Escolar y Desarrollo Profesional (MINEDUC, 2006, 2008).

Se sometió la escala diseñada a juicios de 5 expertos, para ello se tomó contacto en forma individual cada uno de ellos vía e-mail, siguiendo el método de Agregados Individuales (Corral, 2009). En un primer mensaje se les invitó a realizar la evaluación del instrumento, explicándoles cuál era la finalidad de su participación, a aquellos que respondieron afirmativamente a la solicitud se les envío un formato de registro diseñado específicamente para tal efecto que contenía una breve descripción sobre la validación solicitada, una definición de las variables que se buscaba medir, instrucciones y definición de cada criterio considerado para la evaluación.

Optimización del instrumento en base al análisis de los registros realizados por los expertos y, posteriormente, la administración de los ítems a una muestra de sujetos, en forma piloto.

Análisis estadístico para determinar la validez de constructo y fiabilidad del instrumento.

\section{Resultados.}

\subsection{Validación de contenido.}

Para la validación de contenido se optó por seguir un proceso progresivo de optimización de la escala asumiendo lo planteado por Camacho y Sánchez (1997), cuando afirman que «la validación hace referencia al proceso que permite ir depurando, delimitando y perfeccionando cada vez más nuestro instrumento de medida» (p. 99). Este proceso implicó que la escala diseñada fuera enviada a evaluación en dos etapas distintas. Una primera versión fue remitida a dos expertos, una vez recibidos los registros se optimizaron los instrumentos en base al análisis de las anotaciones y recomendaciones, se generó una segunda versión que nuevamente fue enviada al otro grupo de 3 expertos para obtener la valoración final.

La escala de autoevaluación inicialmente tenía 106 indicadores cuyas categorías de respuesta fueron: $1=\mathrm{Nunca} / \mathrm{Nada}-2=\mathrm{Rara}$ vez/Poco $-3=$ A veces/ Ni mucho ni poco 4=Frecuentemente/Bastante y 5= Siempre/ Mucho). Se solicitó a los evaluadores que registraran su valoración por cada uno de ellos considerando los siguientes tres criterios: pertinencia, coherencia y claridad, además de observaciones generales en torno al instrumento. Se llevó a cabo un análisis de cada uno de los registros y a partir de ello se tomaron decisiones sobre la eliminación de aquellos ítems que, a juicio de la mayoría los expertos, no eran coherentes ni pertinentes, y aquellos que no estaban claros se volvieron a redactar.

Finalmente, a partir del análisis sobre los registros de los expertos el instrumento quedó conformado por 90 reactivos distribuidos de la siguiente manera: 
Dimensión Pedagógica: 41 ítems (45,6 \% del instrumento total); Dimensión Social, Ética y Legal: 13 (14,4\% de ítems del instrumento total); Dimensión Técnica: 22 (24,4\% de ítems del instrumento total); Dimensión Gestión Escolar: 6 (6,7 \% de ítems del instrumento total) y Dimensión Desarrollo Profesional: $8(8,9 \%$ de ítems del instrumento total).

\subsection{Validación de constructo y fiabilidad.}

Posterior a la validación de contenido, se aplicó el instrumento a una muestra piloto que consideró 161 estudiantes pertenecientes a las siguientes 9 carreras de Pedagogía de la Facultad de Educación Humanidades de la Universidad del Bío-Bío: Pedagogía en Educación Parvularia, Pedagogía en Educación Básica, Pedagogía en Educación Básica con Especialidad, Pedagogía en Educación Física, Pedagogía en Castellano y Comunicación, Pedagogía en Historia y Geografía, Pedagogía en Educación Matemática, Pedagogía en Inglés y Pedagogía en Ciencias Naturales.

Para la validación de constructo se consideró el análisis factorial (AF), tanto exploratorio como confirmatorio. No obstante, la ratio entre el número de sujetos de la muestra y el número de ítems de la escala impidió la realización del AF sobre el conjunto total de reactivos, por lo que se tomó la decisión de considerar cada una de las cinco dimensiones señaladas anteriormente como subescalas, por ello se presentan los resultados de cada una de ellas en forma separada.

\subsubsection{Análisis Factorial: Dimensión Pedagógica.}

Para la medición de esta dimensión se empleó un cuestionario de 28 ítems tipo Likert con 5 opciones de respuesta (1: Nunca/Nada - 2: Rara vez/Poco - 3: A veces/ Ni mucho ni poco - 4: Frecuentemente/Bastante y 5: Siempre/Mucho). El análisis factorial exploratorio (AFE) determinó la presencia de 6 factores que explican un $63.86 \%$ de la variabilidad total, evidenciándose una dimensión principal, tanto por el número de ítems (13) como el porcentaje de variabilidad explicada (23.71\%). La fiabilidad de estos 28 ítems válidos es muy elevada, alfa de Cronbach $=.937$, altamente significativa con $p<.001$.

Una vez aplicado el instrumento a la muestra definitiva $(N=162)$, se consideró pertinente realizar un análisis factorial confirmatorio (AFC) con la aplicación IBM AMOS 20. La Tabla 1 muestra los índices de ajuste y en ésta se observa que el valor de RMSEA se encuentra muy cerca del .080 y es altamente significativo para una $p<.001$, siendo su IC al 95\%: .076 y .092; por tanto indica un buen ajuste. El resto de índices supera el corte de .800 o se acerca mucho a él. Frente a estos resultados, se puede señalar que el ajuste es suficientemente bueno como para que el resultado del AFC sea fiable.

En la Tabla 2 se presenta los coeficientes ponderados estandarizados de los ítems en los 6 factores teóricos esperados desde el AFE previo. El orden de importancia de los ítems no es exactamente el mismo que en

\begin{tabular}{|c|c|c|c|c|}
\hline Modelo & RMSEA & NFI & IFI & CFI \\
\hline Seis factores & $.084 * * *$ & .787 & .850 & .846 \\
\hline
\end{tabular}

Tabla 1. AFC. Subescala Dimensión Pedagógica. Índices de bondad de ajuste 


\begin{tabular}{|c|c|c|c|c|c|c|c|}
\hline Ítem & Enunciado & \begin{tabular}{|c|} 
Fac. \\
1
\end{tabular} & \begin{tabular}{c|c|c|c|} 
Fac. \\
2
\end{tabular} & \begin{tabular}{c|} 
Fac. \\
$\mathbf{3}$
\end{tabular} & $\begin{array}{c}\text { Fac. } \\
4\end{array}$ & \begin{tabular}{|c|} 
Fac. \\
5
\end{tabular} & $\begin{array}{c}\text { Fac. } \\
6 \\
\end{array}$ \\
\hline DPED 23 & $\begin{array}{l}\text { Aplico estrategias e instrumentos de evaluación soportados por TIC para } \\
\text { valorar el desempeño de los/as estudiantes. }\end{array}$ & .757 & & & & & \\
\hline DPED 17 & $\begin{array}{l}\text { Utilizo criterios (usabilidad, interfaz, accesibilidad e interactividad) para } \\
\text { seleccionar software y recursos tecnológicos posibles para ser usados en } \\
\text { mi sector curricular. }\end{array}$ & .751 & & & & & \\
\hline DPED 22 & $\begin{array}{l}\text { Analizo reflexivamente los resultados y logros alcanzados en } \\
\text { experiencias de aprendizaje desarrolladas con TIC para tomar nuevas } \\
\text { decisiones. }\end{array}$ & .747 & & & & & \\
\hline DPED 25 & $\begin{array}{l}\text { Favorezco la interacción y el trabajo colaborativo en red a través de } \\
\text { estrategias como: resolución de problemas, método de proyecto, } \\
\text { aprendizaje colaborativo, entre otras. }\end{array}$ & .747 & & & & & \\
\hline DPED 26 & Soy capaz de animar y moderar entornos virtuales de aprendizaje & .746 & & & & & \\
\hline DPED 27 & $\begin{array}{l}\text { Favorezco la participación de todos los/as estudiantes en los entornos } \\
\text { virtuales de aprendizaje que organizo. }\end{array}$ & .727 & & & & & \\
\hline DPED 18 & $\begin{array}{l}\text { Comparo herramientas tecnológicas para seleccionar aquellas que } \\
\text { ayudan a atender las necesidades educativas de los/las estudiantes. }\end{array}$ & .722 & & & & & \\
\hline DPED 20 & $\begin{array}{l}\text { Utilizo instrumentos de evaluación para monitorear el aprendizaje de los } \\
\text { estudiantes en entornos de trabajo con TIC. }\end{array}$ & .720 & & & & & \\
\hline DPED 19 & $\begin{array}{l}\text { Examino innovaciones tecnológicas aplicadas en educación (Software } \\
\text { educativos, sitios web, etc.) y recursos digitales existentes en el sistema } \\
\text { escolar e Internet, para seleccionar aquellos que son relevantes en mi } \\
\text { sector curricular y nivel educativo. }\end{array}$ & .715 & & & & & \\
\hline DPED 28 & $\begin{array}{l}\text { Aplico instrumentos de evaluación que me permiten monitorear el } \\
\text { trabajo online en los procesos de aprendizaje de los/as estudiantes. }\end{array}$ & .713 & & & & & \\
\hline DPED 21 & $\begin{array}{l}\text { Aplico instrumentos de evaluación para analizar el resultado de mis } \\
\text { prácticas docentes con TIC. }\end{array}$ & .710 & & & & & \\
\hline DPED 24 & $\begin{array}{l}\text { Utilizo los recursos tecnológicos para recolectar y analizar datos, } \\
\text { interpretar resultados y comunicarlos, con el fin de mejorar los } \\
\text { aprendizajes de los/as estudiantes. }\end{array}$ & .685 & & & & & \\
\hline DPED 16 & $\begin{array}{l}\text { Utilizo diferentes propuestas metodológicas apoyadas en las TIC, como } \\
\text { Webquest, Multimedia Project (MMP), mapas conceptuales, entre otros, } \\
\text { para favorecer experiencias de aprendizaje innovadoras. }\end{array}$ & .668 & & & & & \\
\hline DPED 14 & $\begin{array}{l}\text { Utilizo la tecnología para apoyar estrategias didácticas que den respuesta } \\
\text { a las diversas necesidades de los estudiantes. }\end{array}$ & & .785 & & & & \\
\hline DPED 12 & $\begin{array}{l}\text { Aplico la tecnología para favorecer la creatividad de los estudiantes y } \\
\text { mejorar sus habilidades. }\end{array}$ & & .747 & & & & \\
\hline DPED 15 & $\begin{array}{l}\text { Aplico actividades en las que incorporo recursos TIC como herramientas } \\
\text { de apoyo para favorecer aprendizajes de mi sector curricular. }\end{array}$ & & .734 & & & & \\
\hline DPED 13 & $\begin{array}{l}\text { Oriento a los estudiantes durante el desarrollo de experiencias de } \\
\text { aprendizaje mediadas por las tecnologías. }\end{array}$ & & .717 & & & & \\
\hline DPED 1 & $\begin{array}{l}\text { Identifico los aprendizajes esperados posibles de desarrollar con la } \\
\text { incorporación de las TIC, dentro del programa pedagógico } \\
\text { correspondiente al sector curricular de mi especialidad }\end{array}$ & & .684 & & & & \\
\hline DPED 3 & $\begin{array}{l}\text { Identifico experiencias de aprendizaje de mis sector curricular que } \\
\text { utilicen la tecnología, reconociendo sus fortalezas y desafíos }\end{array}$ & & .615 & & & & \\
\hline DPED 6 & $\begin{array}{l}\text { Defino entornos de trabajo donde los/las estudiantes necesitan utilizar } \\
\text { Internet como medio para abordar los contenidos seleccionados. }\end{array}$ & & & .780 & & & \\
\hline DPED 7 & $\begin{array}{l}\text { Selecciono estrategias de aprendizaje que demandan el uso de } \\
\text { herramientas de productividad (procesador de texto, planillas de cálculo, } \\
\text { software de presentación u otros) }\end{array}$ & & & .711 & & & \\
\hline DPED 5 & $\begin{array}{l}\text { Utilizo algunas estrategias de aprendizaje que requieren del uso de } \\
\text { Internet. }\end{array}$ & & & .600 & & & \\
\hline
\end{tabular}

Tabla 2. Análisis Factorial Confirmatorio. Dimensión Aspectos Pedagógicos 


\begin{tabular}{|c|c|c|c|c|c|c|c|c|}
\hline Ítem & \multicolumn{2}{|l|}{ Enunciado } & Fac. & & & & & \\
\hline DPED 10 & \multicolumn{2}{|c|}{$\begin{array}{l}\text { Empleo herramientas computacionales para el desarrollo de recursos } \\
\text { multimediales como medio de apoyo a las actividades pedagógicas } \\
\text { (creación de páginas web y/o aplicaciones para el desarrollo de éstas). }\end{array}$} & & & & .793 & & \\
\hline DPED 11 & \multicolumn{2}{|c|}{$\begin{array}{l}\text { Diseño objetos de aprendizaje con elementos multimedia } \\
\text { incorporarlos en espacios virtuales de aprendizaje. }\end{array}$} & & & & .765 & & \\
\hline DPED 4 & \multicolumn{2}{|c|}{$\begin{array}{l}\text { Identifico fuentes impresas y/o digitales que contienen información sobre } \\
\text { experiencias educativas que hacen uso de las TIC en mi especialidad. }\end{array}$} & & & & & .698 & \\
\hline DPED 2 & \multicolumn{2}{|c|}{$\begin{array}{l}\text { Identifico las características y finalidad que poseen algunas herramientas } \\
\text { digitales didácticas: Webquest, Wiki, Web Didáctica, Blog u otras. }\end{array}$} & & & & & .589 & \\
\hline DPED 9 & \multicolumn{2}{|c|}{$\begin{array}{l}\text { Utilizo la plantilla de cálculo en la preparación de materiales didácticos } \\
\text { de apoyo a los procesos de enseñanza y aprendizaje, de acuerdo a mi } \\
\text { sector curricular y al nivel donde me desempeño. }\end{array}$} & & & & & & .686 \\
\hline DPED 8 & \multicolumn{2}{|c|}{$\begin{array}{l}\text { Empleo procesadores de texto para producir material didáctico de apoyo a } \\
\text { mis actividades pedagógicas (guías, pruebas, materiales de lectura, entre } \\
\text { otros). }\end{array}$} & & & & & & .466 \\
\hline & \multirow{5}{*}{ Correlación entre factores } & Fac. 1 & -- & .721 & .676 & .756 & \begin{tabular}{|l|l|}
.774 \\
\end{tabular} & .647 \\
\hline & & Fac. 2 & & -- & .863 & \begin{tabular}{|c|}
.613 \\
\end{tabular} & \begin{tabular}{|l|l|}
.837 \\
\end{tabular} & .785 \\
\hline & & Fac. 3 & & & -- & .570 & \begin{tabular}{|l|l|}
.701 \\
\end{tabular} & .753 \\
\hline & & Fac. 4 & & & & -- & .596 & \\
\hline & & Fac. 5 & & & & & -- & .593 \\
\hline
\end{tabular}

Nota: Todos los coeficientes indicados son significativos con $p<.000$

Tabla 2. Análisis Factorial Confirmatorio. Dimensión Aspectos Pedagógicos (continuación)

dicho análisis aunque sí es bastante similar y hay una coincidencia plena en la correspondencia ítem-dimensión. Todos los coeficientes son elevados, de manera que el resultado del AFC confirma la estructura esperada en el cuestionario con los seis factores y, además, con elevadas correlaciones entre ellos.

Posterior a esta nueva validación se calculó la fiabilidad, el valor obtenido en la escala completa con los 28 ítems ha sido .951 [IC al 95\%: .939- .961], que es por tanto muy elevado. Por factores, el alfa de Cronbach de cada uno de ellos ha sido el siguiente: Factor 1(13 ítems, .934); Factor 2 (6 ítems, .860), Factor 3 (3 ítems, .730), Factor 4 ( 2 ítems, . 755), Factor 5 (2 ítems, .578) y factor 6 (2 ítems, $.482)$.

En consecuencia, la fiabilidad es muy buena para 4 factores y la puntuación total, mientras que es un poco pobre en los dos últimos factores, aunque con tan pocos ítems se puede decir que tiene bastante mérito.

\subsubsection{Análisis Factorial: Dimensión Aspectos Sociales, Éticos y Legales.}

Este constructo se evaluó con una subescala que contenía 13 ítems también tipo Likert con 5 opciones de respuesta (1: Nunca/ Nada - 2: Rara vez/Poco - 3: A veces/Ni mucho ni poco- 4: Frecuentemente/Bastante y 5: Mucho/Siempre). El AFE realizado inicialmente determinó la existencia de tres componentes que explican un $71.78 \%$ de la variabilidad total. En cuanto a la fiabilidad obtenida fue bastante elevada (alfa de Cronbach=.926, IC al 95\%: .97 - .942) y altamente significativa con $p<.001$.

$\mathrm{Al}$ igual que en el caso anterior, se realizó el correspondiente análisis factorial confirmatorio, el valor de RMSEA está cerca del .080 siendo su IC al 95\%: .076 - .103, 


\begin{tabular}{|c|c|c|c|c|}
\hline Modelo & RMSEA & NFI & IFI & CFI \\
\hline Tres factores & $.094 * * *$ & .888 & .929 & .927 \\
\hline$* * *$ Significativo con $p<.000$
\end{tabular}

Tabla 3. AFC. Subescala Dimensión Aspectos Sociales, Éticos y Legales. Índices de bondad de ajuste

altamente significativo para un $p<.001$; por tanto indica un buen ajuste. El resto de índices supera claramente el corte de .800 e incluso el valor 900 (ver Tabla 3 ).

Los coeficientes ponderados estandarizados de los ítems en los 3 factores teóricos previstos por el AFE previo se pueden observar en la Tabla 4. El orden de importancia de los ítems no es exactamente el mismo, pero si es muy similar y con una coincidencia plena en la correspondencia ítem-dimensión. Todos los coeficientes son muy altos, de manera que este AFC confirma la estructura esperada en la escala con los tres factores. Asimismo, se observa que el modelo obtiene elevadas correlaciones entre todos los factores.

Una vez confirmada la nueva validación de la escala, se sigue con el cálculo de la fiabilidad, el valor del alfa de Cronbach obtenido en la escala completa con los 13 ítems ha sido .922, IC al 95\%: .871 - .920, por tanto muy elevado. La fiabilidad por factores fue la siguiente: Factor 1(5 ítems, .897); Factor 2 (5 ítems, .882); Factor 3 (3 ítems, .778).

\subsubsection{Análisis Factorial: Dimensión Aspectos Técnicos.}

La subescala diseñada contiene 19 ítems en formato Likert con 5 opciones de respuesta (1: Nada/Nunca - 2: Rara vez/Poco - 3: A veces/ Ni mucho ni poco - 4: Frecuentemente/Bastante y 5: Mucho/ Siempre). El AFE realizado inicialmente determinó la necesidad prescindir de tres ítems para obtener un resultado válido, decisión que consiguió una variabilidad explicada del $62.91 \%$ con 4 componentes y con 19 ítems. La fiabilidad de este conjunto de reactivos también ha sido muy elevada (alfa de Cronbach $=.911$, IC 95\% de: $.888-$ .930) $\operatorname{con} p<.001$.

$\mathrm{Al}$ igual que en las dimensiones anteriores, se procedió a realizar el análisis factorial confirmatorio. El valor de RMSEA está cerca del .080 siendo su IC al 95\%: .082 - .105, altamente significativo para un $p<.001$, lo que indica un ajuste aceptable. El resto de índices supera el corte de .800 o se aproxima a él, por lo que se concluye que el ajuste permite que el resultado del AFC sea fiable (ver Tabla 5).

En la Tabla 6 se pueden observar los valores de los coeficientes ponderados estandarizados de los ítems en los cuatro factores teóricos previstos por el AFE. Como en los casos anteriores, y aunque el orden de importancia de los ítems no es el mismo, sí que es semejante y con plena coincidencia en la correspondencia ítem-factor. Los coeficientes son altos, de manera que este AFC confirma la estructura esperada en el cuestionario con los cuatro factores, con excepción del ítem 60 cuyo peso en el Factor 3 se ha reducido bastante hasta el punto de que se decide prescindir de él. Asimismo, este AFC también muestra que el modelo incluye notables correlaciones entre los factores.

Posteriormente se continuó con el cálculo de la fiabilidad para los 18 ítems admitidos en el modelo (se excluye el ítem 60, mencionado anteriormente). La escala completa tuvo un 


\begin{tabular}{|c|c|c|c|c|}
\hline Ítem & Enunciado & \begin{tabular}{c|} 
Fact. \\
1
\end{tabular} & \begin{tabular}{c|} 
Fact. \\
2
\end{tabular} & $\begin{array}{c}\text { Fact. } \\
\mathbf{3}\end{array}$ \\
\hline \begin{tabular}{|c|} 
ETICO \\
36
\end{tabular} & $\begin{array}{l}\text { Incorporo a mis pares y otros actores del entorno educativo en diálogos sobre el uso } \\
\text { e impacto de las TIC en educación. }\end{array}$ & .873 & & \\
\hline $\begin{array}{l}\text { ETICO } \\
35\end{array}$ & $\begin{array}{l}\text { Comento con mis pares y otros miembros de la comunidad educativa las inquietudes } \\
\text { y certezas que poseo sobre el uso e impacto de las TIC en el desarrollo de la } \\
\text { sociedad. }\end{array}$ & .795 & & \\
\hline \begin{tabular}{c|} 
ETICO \\
31
\end{tabular} & $\begin{array}{l}\text { Dialogo con mis pares sobre las posibilidades que ofrecen las TIC para favorecer la } \\
\text { interacción comunicativa y la construcción del conocimiento. }\end{array}$ & .792 & & \\
\hline \begin{tabular}{|c|} 
ETICO \\
29
\end{tabular} & Analizo con mis pares el impacto de las TIC en diferentes ámbitos de la sociedad. & .770 & & \\
\hline $\begin{array}{c}\text { ETICO } \\
30\end{array}$ & $\begin{array}{l}\text { Promuevo espacios de conversación con los/as estudiantes donde se analiza el } \\
\text { impacto de las TIC en diferentes ámbitos de la sociedad, de acuerdo a su nivel de } \\
\text { desarrollo. }\end{array}$ & .760 & & \\
\hline $\begin{array}{c}\text { ETICO } \\
41\end{array}$ & $\begin{array}{l}\text { Dialogo con otros actores de la comunidad escolar sobre la importancia del uso ético } \\
\text { y legal de las aplicaciones informáticas e informacionales, disponibles en sus } \\
\text { diferentes formatos. }\end{array}$ & & .878 & \\
\hline \begin{tabular}{|c|} 
ETICO \\
39
\end{tabular} & $\begin{array}{l}\text { Dialogo con los estudiantes sobre el actuar ético y legal en lo que respecta al empleo } \\
\text { de la tecnología, de la información y de software. }\end{array}$ & & .813 & \\
\hline $\begin{array}{c}\text { ETICO } \\
40\end{array}$ & $\begin{array}{l}\text { Defiendo la importancia de utilizar software, aplicaciones e información disponible } \\
\text { en la red, en el marco de los aspectos éticos y legales. }\end{array}$ & & .798 & \\
\hline $\begin{array}{c}\text { ETICO } \\
38\end{array}$ & $\begin{array}{l}\text { Considero los aspectos legales y éticos asociados a la información y comunicación } \\
\text { en cada una de las actividades donde hago uso de la tecnología. }\end{array}$ & & .703 & \\
\hline $\begin{array}{c}\text { ETICO } \\
37\end{array}$ & $\begin{array}{l}\text { Distingo los aspectos legales y éticos asociados a la información digital (privacidad, } \\
\text { propiedad intelectual, seguridad de la información, licenciamiento de software, etc.) }\end{array}$ & & .667 & \\
\hline
\end{tabular}

Tabla 4. Análisis Factorial Confirmatorio. Subescala Dimensión Aspectos Sociales, Éticos y Legales

\begin{tabular}{|c|c|c|c|c|c|}
\hline Ítem & \multicolumn{2}{|l|}{ Enunciado } & $\begin{array}{c}\text { Fact. } \\
1\end{array}$ & \begin{tabular}{c|c} 
Fact. \\
2
\end{tabular} & $\begin{array}{l}\text { Fact. } \\
3\end{array}$ \\
\hline $\begin{array}{c}\text { ETICO } \\
33\end{array}$ & \multicolumn{2}{|c|}{$\begin{array}{l}\text { Distingo las posibilidades que ofrecen los recursos tecnológicos para facilitar el } \\
\text { aprendizaje de los/as estudiantes, de acuerdo a su nivel educativo. }\end{array}$} & & & .857 \\
\hline $\begin{array}{c}\text { ETICO } \\
34 \\
\end{array}$ & \multicolumn{2}{|c|}{$\begin{array}{l}\text { Reconozco la importancia de ofrecer igualdad de oportunidades para que todos los/as } \\
\text { estudiantes tengan acceso a los recursos tecnológicos que utilizo en las clases. }\end{array}$} & & & .695 \\
\hline $\begin{array}{c}\text { ETICO } \\
32\end{array}$ & \multicolumn{2}{|c|}{$\begin{array}{l}\text { Utilizo los recursos tecnológicos para facilitar el aprendizaje de los estudiantes en } \\
\text { diferentes entornos de trabajo, respetando sus características individuales y de acuerdo } \\
\text { a su nivel educativo. }\end{array}$} & & & .671 \\
\hline & \multirow{2}{*}{ Correlación entre factores } & Fact. 1 & --- & .718 & .719 \\
\hline & & Fact. 2 & & --- & .621 \\
\hline
\end{tabular}

Tabla 4. Análisis Factorial Confirmatorio. Subescala Dimensión Aspectos Sociales, Éticos y Legales (continuación)

alfa de Cronbach de .915, IC al 95\%: .895 933. La consistencia interna de cada uno de los factores fue la siguiente: Factor 1(6 ítems, .823); Factor 2 (6 ítems, .843); Factor 3 (4 ítems, .746) y Factor 4 (2 ítems, .894).

En virtud a los resultados, se puede decir que la fiabilidad es buena, tanto para los factores como para la puntuación total.

\subsubsection{Análisis Factorial: Dimensión Gestión Escolar.}

Esta dimensión fue evaluada con una escala tipo Likert que contiene 6 ítems con las mismas 5 opciones de respuesta que las anteriores (1: Nunca/Nada - 2: Rara vez/Poco - 3: A veces/Ni mucho ni poco- 4: Frecuentemente/ Bastante y 5: Mucho/Siempre). El AFE 


\begin{tabular}{|}
\begin{tabular}{|c|c|c|c|c|}
\hline Modelo & RMSEA & NFI & IFI & CFI \\
\hline Cuatro factores & $.093 * * *$ & .756 & .826 & .821 \\
\hline$* * *$ Significativo con $p<.000$
\end{tabular} \\
\hline
\end{tabular}

Tabla 5. AFC. Cuestionario de la dimensión Aspectos Técnicos. Índices de bondad de ajuste

\begin{tabular}{|c|c|c|c|c|c|}
\hline Ítem & Enunciado & $\begin{array}{c}\text { Fact } \\
1\end{array}$ & $\begin{array}{c}\text { Fact. } \\
2\end{array}$ & Fact & $\begin{array}{c}\text { Fact } \\
4\end{array}$ \\
\hline $\begin{array}{c}\text { TEC } \\
52\end{array}$ & $\begin{array}{l}\text { Empleo en las presentaciones diversos recursos tecnológicos como imágenes, animaciones, } \\
\text { hipervínculos u otras alternativas. }\end{array}$ & .770 & & & \\
\hline $\begin{array}{c}\text { TEC } \\
51\end{array}$ & Utilizo algún software de presentación para exponer información que quiero comunicar. & .687 & & & \\
\hline $\begin{array}{c}\text { TEC } \\
55\end{array}$ & Empleo los recursos disponibles en Internet para buscar la información que requiero. & .687 & & & \\
\hline $\begin{array}{c}\text { TEC } \\
53\end{array}$ & Diseño presentaciones que permiten alcanzar impacto en el mensaje que quiero comunicar. & .640 & & & \\
\hline $\begin{array}{c}\text { TEC } \\
59\end{array}$ & $\begin{array}{l}\text { Utilizo una cuenta de correo electrónico para comunicarme con otros a través del envío y } \\
\text { recepción de mensajes. }\end{array}$ & .633 & & & \\
\hline $\begin{array}{c}\text { TEC } \\
48\end{array}$ & $\begin{array}{l}\text { Empleo recursos del procesador de textos como tablas, cuadros e imágenes dentro del } \\
\text { documento. }\end{array}$ & .612 & & & \\
\hline $\begin{array}{c}\text { TEC } \\
46\end{array}$ & $\begin{array}{l}\text { Actualizo mis conocimientos respecto del desarrollo de las tecnologías informáticas y sus } \\
\text { nuevas aplicaciones en educación. }\end{array}$ & & .763 & & \\
\hline $\begin{array}{c}\text { TEC } \\
47\end{array}$ & $\begin{array}{l}\text { Utilizo el procesador de textos para la creación de documentos dejándolos aptos para su } \\
\text { distribución. }\end{array}$ & & .754 & & \\
\hline \begin{tabular}{c|} 
TEC \\
43
\end{tabular} & Empleo el sistema operativo para administrar y gestionar archivos, carpetas y aplicaciones. & & .726 & & \\
\hline $\begin{array}{c}\text { TEC } \\
44\end{array}$ & Gestiono el uso de recursos en la red local (impresoras, carpetas y archivos, configuración). & & .675 & & \\
\hline $\begin{array}{c}\text { TEC } \\
45\end{array}$ & $\begin{array}{l}\text { Aplico medidas de seguridad y prevención de riesgos en la operación de equipos tecnológicos y } \\
\text { la salud de las personas. }\end{array}$ & & .667 & & \\
\hline $\begin{array}{c}\text { TEC } \\
42\end{array}$ & $\begin{array}{l}\text { Identifico conceptos y componentes básicos asociados a la tecnología informática en ámbitos } \\
\text { como hardware, software y otros. }\end{array}$ & & .569 & & \\
\hline $\begin{array}{c}\text { TEC } \\
58\end{array}$ & $\begin{array}{l}\text { Uso información textual y gráfica obtenida desde Internet en la preparación de diversos tipos de } \\
\text { documentos con software de productividad, citando las fuentes correspondientes }\end{array}$ & & & .730 & \\
\hline $\begin{array}{c}\text { TEC } \\
56\end{array}$ & $\begin{array}{l}\text { Soy capaz de acceder, buscar y recuperar información utilizando alguna plataforma de Internet, } \\
\text { como Google Drive, Dropbox, Amazon Cloud Drive, entre otras. }\end{array}$ & & & .682 & \\
\hline $\begin{array}{c}\text { TEC } \\
57\end{array}$ & $\begin{array}{l}\text { Sé realizar videoconferencias por IP (NetMeeting, Messenger, Skype, etc.) a través de Internet } \\
\text { pata comunicarme con mis contactos. }\end{array}$ & & & .633 & \\
\hline $\begin{array}{c}\text { TEC } \\
54\end{array}$ & $\begin{array}{l}\text { Reconozco el significado de algunos conceptos utilizados en la red, por ejemplo: Internet, } \\
\text { WWW, buscadores, hipertextos, Microblogging, Lectores RRS, Marcadores Sociales, } \\
\text { groupware, etc. }\end{array}$ & & & .587 & \\
\hline $\begin{array}{c}\text { TEC } \\
60 \\
\end{array}$ & $\begin{array}{l}\text { Diseño y publico información en la red utilizando diferentes formatos: Páginas Web, Blogs, } \\
\text { Foros, Plataformas Virtuales, etc. }\end{array}$ & & & .364 & \\
\hline $\begin{array}{c}\text { TEC } \\
49\end{array}$ & $\begin{array}{l}\text { Utilizo la planilla de cálculo para procesar datos e informar resultados de manera numérica y } \\
\text { gráfica. }\end{array}$ & & & & .932 \\
\hline $\begin{array}{c}\text { TEC } \\
50\end{array}$ & Aplico funciones matemáticas y lógicas utilizando fórmulas básicas en una planilla de cálculo. & & & & .867 \\
\hline & \multirow{3}{*}{ Correlación entre factores } & --- & .751 & .747 & .507 \\
\hline & & & --- & .719 & .652 \\
\hline & & & & --- & .518 \\
\hline
\end{tabular}

Tabla 6. Análisis Factorial Confirmatorio. Subescala Dimensión Aspectos Técnicos. 
realizado inicialmente determinó la presencia de un único factor con los 6 ítems que explica el $57.13 \%$ de la variabilidad total, y aunque no es un resultado notable sí es al menos suficiente para admitir la validez de esta subescala. En cuanto a la fiabilidad y, a pesar de que el número de ítems es bajo, se ha obtenido un coeficiente de consistencia interna muy bueno (alfa de Cronbach $=.846$, IC al 95\%: .805- .880), altamente significativo con $p<.001$.

$\mathrm{Al}$ igual que en las dimensiones anteriores, se llevó a cabo el análisis factorial confirmatorio. El valor de RMSEA se aleja del valor ideal siendo su IC al 95\%: .107 - .115, aunque es altamente significativo para un $p<.001$. Por su parte, el resto de índices supera claramente el corte de .800 e incluso el de .900. Aunque el primero de ellos no cumple el requisito se puede deber a la existencia de un solo factor, los demás índices lo hacen sobradamente por lo que se considera que el ajuste logrado permite que el resultado del AFC sea fiable (ver Tabla 7).

Los valores de los coeficientes ponderados estandarizados de los ítems en este único factor, previstos por el AFE, se presentan en la Tabla 8. El orden de importancia de los ítems no es el mismo que en el análisis factorial exploratorio, pero sí que es semejante y se mantiene la alta pertenencia de cada uno de ellos al factor predefinido. En consecuencia, el AFC confirma la estructura esperada en el cuestionario para esta dimensión.

Después de la validación de esta subescala se continúa con el cálculo de la fiabilidad y el valor obtenido es .833 [IC al 95\%: .791 - .869], que es muy bueno.

\subsubsection{Análisis Factorial: Dimensión Desarrollo Profesional.}

Para este constructo se dispuso de una subescala con 7 ítems de tipo Likert con sus 5 opciones de respuesta (1: Nunca/Nada - 2: Rara vez/Poco - 3: A veces/Ni mucho ni poco4: Frecuentemente / Bastante y 5: Mucho/ Siempre). El AFE realizado inicialmente determinó la existencia de 2 factores, con 7 reactivos, que explicarían en $77.32 \%$ de la variabilidad total. El grado de fiabilidad logrado por estos 7 ítems es elevado (alfa de Cronbach $=.907$, IC al 95\%: $.884-.928$ ), altamente significativo $\operatorname{con} p<.001$.

Se realizó el correspondiente análisis factorial confirmatorio. El valor de RMSEA contiene el punto ideal .080, siendo su IC al 95\%:.078 - .144 y es altamente significativo para una $p<.001$, por lo que indica un buen ajuste. El resto de índices supera claramente el corte de .800 e incluso el valor .900 , de modo que con estos resultados se concluye que el ajuste es bueno y que el resultado del AFC sea fiable (ver Tabla 9).

En la Tabla 10 se presentan los coeficientes ponderados estandarizados de los ítems en los 2 factores teóricos previstos por el AFE. Una vez más el orden de importancia de los ítems no es exactamente el mismo, pero es similar y hay coincidencia plena en la correspondencia ítem-dimensión. Todos los coeficientes son muy elevados, de manera

\begin{tabular}{|l|c|c|c|c|}
\hline \multicolumn{1}{|c|}{ Modelo } & RMSEA & NFI & IFI & CFI \\
\hline Un factor & $.149 * * *$ & .883 & .905 & .902 \\
\hline *** Significativo con $p<.000$ \\
\hline
\end{tabular}

Tabla 7. AFC. Subescala dimensión Gestión Escolar. Índices de bondad de ajuste

Píxel-Bit. Revista de Medios y Educación. No 48 Enero 2016. ISSN: 1133-8482. e-ISSN: 2171-7966. doi: http://dx.doi.org/10.12795/pixelbit.2016.i48.14 


\begin{tabular}{|c|l|c|}
\hline Ítem & \multicolumn{1}{|c|}{ Enunciado } & $\begin{array}{c}|c| \\
\mathbf{1}\end{array}$ \\
\hline GEST 64 & $\begin{array}{l}\text { Utilizo los recursos tecnológicos existentes en el centro educativo para apoyar las tareas } \\
\text { administrativas y pedagógicas. }\end{array}$ & .758 \\
\hline GEST 66 & $\begin{array}{l}\text { Soy capaz de proporcionar información relevante a la comunidad educativa haciendo uso de las } \\
\text { tecnologías. }\end{array}$ & .758 \\
\hline GEST 61 & $\begin{array}{l}\text { Recurro a software de productividad y/ aplicaciones de Internet para elaborar material } \\
\text { administrativo relacionado con mi función docente (cartas a apoderados, informes de notas, } \\
\text { planificaciones, trípticos, afiches, etc.) }\end{array}$ & .677 \\
\hline GEST 65 & $\begin{array}{l}\text { Elaboro documentos propios de la actividad administrativa del establecimiento (trípticos, afiches, } \\
\text { comunicados, etc.) haciendo uso de las tecnologías. }\end{array}$ & .642 \\
\hline GEST 62 & $\begin{array}{l}\text { Utilizo los recursos informáticos para elaborar o administrar bases de datos con información } \\
\text { sobre los7as estudiantes para apoyar los procesos administrativos. }\end{array}$ & .620 \\
\hline GEST 63 & $\begin{array}{l}\text { Empleo los servicios que ofrece Internet (correo electrónico, plataformas virtuales, redes } \\
\text { sociales, etc.) para establecer un contacto permanente con los miembros de la comunidad } \\
\text { educativa. }\end{array}$ & .605 \\
\hline Nota: Todos los coeficientes indicados son significativos con $p<.000$ \\
\hline
\end{tabular}

Tabla 8. Análisis Factorial Confirmatorio. Dimensión Gestión Escolar.

\begin{tabular}{|c|c|c|c|c|}
\hline Modelo & RMSEA & NFI & IFI & CFI \\
\hline Dos factores & $.095 * * *$ & .935 & .954 & .953 \\
\hline *** Significativo con $p<.000$ & \\
\hline
\end{tabular}

Tabla 9. AFC. Dimensión Desarrollo Profesional. Índices de bondad de ajuste

\begin{tabular}{|c|c|c|c|}
\hline Ítem & Enunciado & $\begin{array}{c}\text { Fact. } \\
1\end{array}$ & $\begin{array}{c}\text { Fact } \\
2\end{array}$ \\
\hline DESPRO 72 & $\begin{array}{l}\text { Comparto en la red algunos materiales digitales que he creado o mis ideas y } \\
\text { experiencias en torno a la utilización de recursos TIC. }\end{array}$ & .842 & \\
\hline DESPRO 70 & $\begin{array}{l}\text { Participo en espacios de reflexión e intercambio de experiencias sobre el diseño e } \\
\text { implementación de actividades pedagógicas con TIC. }\end{array}$ & .805 & \\
\hline DESPRO 73 & $\begin{array}{l}\text { Participo en diferentes instancias relacionadas con el desarrollo de la informática } \\
\text { educativa (Congresos, foros, Seminarios, muestras, etc.) }\end{array}$ & .794 & \\
\hline DESPRO 71 & $\begin{array}{l}\text { Utilizo las herramientas de comunicación provistas por Internet para el intercambio de } \\
\text { experiencias con otras unidades educativas y para comunicarme con pares que pueden } \\
\text { ayudar a mi labor docente. }\end{array}$ & .745 & \\
\hline DESPRO 67 & $\begin{array}{l}\text { Accedo a fuentes de información para actualizarme en informática educativa, tales } \\
\text { como: revistas electrónicas, portales educativos, participación en listas de interés. }\end{array}$ & & .806 \\
\hline DESPRO 69 & $\begin{array}{l}\text { Busco información actualizada sobre las tecnologías aplicadas a educación y sobre } \\
\text { experiencias innovadoras para fundamentar la adecuación de mis prácticas educativas. }\end{array}$ & & .789 \\
\hline DESPRO 68 & $\begin{array}{l}\text { Utilizo los portales educativos nacionales e internacionales como espacio de acceso a } \\
\text { recursos digitales validados por expertos que pueden enriquecer mi labor docente. }\end{array}$ & & .779 \\
\hline & Correlación entre factores & --- & .731 \\
\hline
\end{tabular}

Tabla 10. Análisis Factorial Confirmatorio. Dimensión Desarrollo Profesional. 
que el AFC confirma la estructura esperada en esta subescala. Así mismo, el AFC muestra que el modelo incluye una elevada correlación entre los factores.

Una vez realizada esta nueva validación, se prosigue con el estudio de la fiabilidad y el valor del coeficiente alfa de Cronbach obtenido en esta subescala completo con los 7 ítems ha sido .888, IC al 95\%: .861 - .912. Por factores, el coeficiente de fiabilidad de cada uno de ellos es el siguiente: Factor 1(4 ítems, .872); Factor 2 (3 ítems, .833).

En consecuencia, se puede decir que la fiabilidad de esta subescala es muy buena, tanto para los 2 factores como para la puntuación total.

\section{Discusión y conclusiones.}

La competencia digital del docente debe ser abordada desde las distintas dimensiones que la componen, es decir, desde una perspectiva holística y orientada más al «saber ser y hacer con las TIC» que al «saber sobre las TIC», porque hoy en día no es suficiente que el profesorado posea amplios conocimientos sobre las posibilidades educativas que las herramientas tecnológicas y digitales ofrecen, ya que lo relevante, a nuestro juicio, es la capacidad que tenga el docente para ejercer una acción didáctica con ellas, pues la incorporación de las TIC en el proceso de enseñanza y aprendizaje no sólo implica que el docente deba reflexionar en torno al qué, cómo, cuándo y para qué utilizarlas, sino que se requiere que este actor sea capaz de replantearse nuevas formas de enseñar y aprender, de decidir apropiarse de ellas y capacitarse para utilizarlas convenientemente, es decir, utilizarlas para realizar una enseñanza más constructiva, innovadora y de calidad aprovechando todas las ventajas que las herramientas tecnológicas y digitales pueden ofrecer.

El conjunto de desafíos al que se ve enfrentado actualmente el profesorado, debe ser valorado por las instituciones de educación superior como una gran oportunidad que pueden contribuir a renovar los programas de formación inicial docente, pues, algunos autores enfatizan en que esta etapa sería el momento propicio para desarrollar la competencia digital docente (De Saint, 2008; Flores, 2014; Suárez, Almerich, Díaz \& Fernández, 2011; Vaillant, 2013), pero bajo un modelo formativo que contemple una perspectiva global, por cuanto los programas de formación de docentes no pueden continuar estando centrados exclusivamente en la dimensión técnica e instrumental de las TIC, como ha sucedido hasta el momento (Cabero,2004; Valverde, Garrido \& Fernández, 2010).

En virtud a lo anterior, consideramos que para renovar los planes de formación inicial docente en lo que respecta a las TIC, se necesita contar con información válida y fiable sobre los niveles de desempeño de las y los futuros maestros en cada una de las dimensiones de la competencia digital, ya que de este modo se podrían tomar decisiones fundamentadas empíricamente sobre las intervenciones necesarias de realizar a nivel curricular, metodológico o evaluativo, así como también en lo que respecta al perfeccionamiento de los docentes formadores. Por tal razón, como una primera etapa, se ha querido diseñar un instrumento válido y fiable, que contribuya a la obtención de dicha información a partir de la autovaloración que realizan las y los estudiantes.

El proceso de diseño y validación del instrumento aquí presentado, permite 
evidenciar que cada una de las dimensiones que conforman la Escala de Autovaloración de competencias digitales para estudiantes de Pedagogía, ha demostrado de manera suficiente tanto su validez estructural como su fiabilidad. No obstante a ello, consideramos que dentro de las principales limitaciones que este instrumento presenta se encuentra la reducida cantidad de la muestra, por cuanto solo se validó en un contexto específico. Asimismo, destacamos que al estar centrado exclusivamente en la percepción de los discentes sobre su competencia digital, la información registrada podría tener ciertos sesgos, propios de un proceso subjetivo, lo que en alguna medida podría haber influido en los resultados obtenidos. Por ello, una de las principales proyecciones que proponemos es la continuidad en la aplicación y validación de este instrumento en otros contextos e idealmente con una cantidad mayor de muestra.

\section{Fuente de financiación.}

El trabajo aquí presentado se enmarca dentro de la tesis doctoral titulada «Factores que inciden en la valoración de estudiantes de Pedagogía sobre sus competencias digitales: El caso de la Universidad del BíoBío (Chile)», desarrollada en el Programa de Doctorado en Investigación Educativa: Enseñanza y Aprendizaje de la Universidad de Alicante, España. Esta tesis ha sido financiada por el Ministerio de Educación de Chile y la Universidad del Bío-Bío, a través del Proyecto MECESUP UBB: 0704-2011.

\section{Referencias bibliográficas.}

Adell, J. (2010, Octubre). Dimensiones de la competencia digital. Parte II de la Ponencia La Competencia Digital. XXIV Jornadas Pedagógicas de Barakaldo. Disponible en https://goo.gl/gTqUFk

Area, M. (2010). ¿Por qué formar en competencias informacionales y digitales en la educación superior?. Revista de Universidad y Sociedad del Conocimiento (RUSC), 7(2). Recuperado de http://goo.gl/ $\mathrm{h} 2 \mathrm{vyrX}$

Arras, A.M., Torres, C. \& García, A.M. (2011). Competencias en Tecnologías de Información y Comunicación (TIC) de los estudiantes universitarios. Revista Latina de Comunicación Social, 66, 130-152. doi:10.4185/RLCS-66-2011-927-130-152/ CrossReflink

Brun, M. (2011). Las tecnologías de la información y comunicaciones en la Formación Inicial Docente en América Latina. Santiago de Chile: Cepal (Serie políticas sociales, $\mathrm{n}^{\circ}$ 172). Recuperado de http://goo.gl/zCnwSI

Cabero, J. (2004). Formación del profesorado. El gran caballo de batalla. Comunicación y Pedagogía. Tecnologías y Recursos didácticos, 195, 27-31. Recuperado de http://goo.gl/6wztwb

Cabero, J., Llorente, M.C. \& Marín, V. (2010). Hacia el diseño de un instrumento de diagnóstico de «competencias tecnológicas del profesorado» universitario. Revista Iberoamericana de Educación, 52(7), 1-12. Recuperado de http://goo.gl/mbRWdM

Cabero, J. \& Marín, V. (2014). Miradas sobre la formación del profesorado en tecnologías de información y comunicación (TIC). Enl@ce: Revista Venezolana de Información de, Tecnología y Conocimiento, 11(2), 11-24. 
Camacho, C. \& Sánchez, E.F. (1997). Psicometría. Sevilla: Kronos.

Climént, J. (2010). Reflexiones sobre la educación basada en competencias. Revista Complutense de Educación, 21(1), 91-106.

Corral, Y. (2009). Validez y confiabilidad de los instrumentos de investigación para la recolección de datos. Revista Ciencias de la Educación, 19(33), 228-247. Recuperado de http://goo.gl/xmIe70

De Saint, D. (27 de Octubre de 2008). Futuros profesores: ¿por qué formar en competencias digitales? [Blog]. Recuperado de http://goo.gl/2nYaew

Diario Oficial de la Unión Europea (2006). Recomendación del Parlamento Europeo y del Consejo de 18 de diciembre de 2006 sobre las competencias clave para el aprendizaje permanente. Recuperado de http:/goo.gl/ $1 \mathrm{k} 3 \mathrm{DLc}$

Flores, C. (2014). Competencia digital docente: desempeños didácticos en la formación inicial del profesorado. Revista Científica de Educación y Comunicación. Hachetetepe, 9, 55-70. Recuperado de http:// goo.gl/N27Rsz

García, A. (2011). Integración de las TIC en la docencia universitaria. España: Netbiblo.

Gisbert, M., Espuny, C. \& González, J. (2011). INCOTIC. Una herramienta para la @utoevaluación diagnóstica de la competencia digital en la universidad. Profesorado. Revista de currículum y formación del profesorado, 15 (1), 75-90. Recuperado de http://goo.gl/p0IBt7

Gutiérrez, I. (2014). Perfil del profesor universitario español en torno a las competencias en tecnologías de la información y de la comunicación. Pixel-Bit. Revista de Medios y Educación, 44, 51-65. doi: http://dx.doi.org/10.12795/ pixelbit.2014.i44.04

International Society for Technology in Education (2008). National Educational Technology Standards for Teachers (NETST), Second Edition. ISTE®. (Versión en español). Recuperado de http://goo.gl/ Xt9CET

Mengual, S. \& Roig, R. (2012). La enseñanza y las competencias TIC en el contexto universitario. En Sandoval, Y., Arenas, A., López, E., Cabero, J. \& Aguared, J.I. (Coords.). Las tecnologías de la información en contextos educativos (pp. 17-24). Colombia: Universidad Santiago de Cali. Recuperado de http://goo.gl/zRpaHu

Ministerio de Educación de Chile (2006). Estándares TIC para la FID Competencias para la formación Docente. (Primera Parte). Santiago de Chile. Recuperado de www.enlaces.cl

Ministerio de Educación de Chile [ENLACES] (2008). Estándares en Tecnología de la Información y la Comunicación para la Formación Inicial Docente. Una propuesta en el contexto chileno. Recuperado de http://goo.gl/PqYtiD

Ministerio de Educación Nacional Colombia (2008). Ruta de Apropiación de TIC para el Desarrollo Profesional Docente RUTA. Recuperado de http://goo.gl/rXQxzo

Prendes, M.P. (Dir.) (2010). Competencias TIC para la docencia en la Universidad Pública española. Indicadores y propuestas para la definición de buenas prácticas. Programa de estudio y Análisis. Informe del proyecto EA2009-0133 de la Secretaría de Estado de universidades e Investigación. Recuperado de http://goo.gl/L1BRS4

Prendes, M.P. \& Castañeda, L. (2010). Enseñanza superior, profesores y TIC. Estrategias de evaluación, investigación e 
innovación educativa. Sevilla: Editorial MAD, S.L.

Prendes, M.P. \& Gutiérrez, I. (2013). Competencias tecnológicas del profesorado en las universidades españolas. Revista de Educación, 361, 196-222. doi:10.4438/1988592X-RE-2011-361-140

Suárez, J.M., Almerich, G., Díaz, I. \& Fernández, R. (2012). Competencias del profesorado en las TIC. Influencia de factores personales y contextuales. Universitas Psychologica, 11(1), 293-309. Recuperado de http://goo.gl/hKGyoH

UNESCO (2008). Norma de Estándares de competencias TIC para docentes. París: Organización de las Naciones Unidas para la Educación, la Ciencia y la Cultura. Recuperado de http://goo.gl/Ww5VCV

Vaillant, D. (2013). Integración de TIC en los sistemas de formación docente inicial y continua para la Educación Básica en América Latina. Argentina: Fondo de las Naciones Unidas para la Infancia (UNICEF). Recuperado de http://goo.gl/Vg8lcP

Valverde, J., Garrido, M.C. \& Fernández, R. (2010). Enseñar y aprender con tecnologías: Un modelo teórico para las Buenas Prácticas con TIC. TESI, 11 (3), 203-229. Recuperado de http://goo.gl/NhAV6L

Fecha de recepción: 07-09-2015

Fecha de evaluación: 24-09-2015

Fecha de aceptación: 15-10-2015 